\title{
El efecto de la covid-19 en la impartición de cursos de matemáticas: evidencia experimental en una macrouniversidad de México
}

The effect of COVID-19 on the delivery of mathematics courses: experimental evidence in a macrouniversity in Mexico

O efeito do COVID-19 no ensino de cursos de matemática: evidências experimentais em uma macrouniversidade no México

\author{
Elías Alvarado Lagunas \\ Universidad Autónoma de Nuevo León, México \\ eliaxalvarado@gmail.com \\ https://orcid.org/0000-0002-2751-7718 \\ Dionicio Morales Ramírez \\ Universidad Autónoma de Tamaulipas, México \\ dionicio.morales@gmail.com \\ https://orcid.org/0000-0002-6859-6547 \\ Jeyle Ortiz Rodríguez \\ Universidad Autónoma de Nuevo León, México \\ jeyleortiz@gmail.com \\ https://orcid.org/0000-0001-6220-7742
}




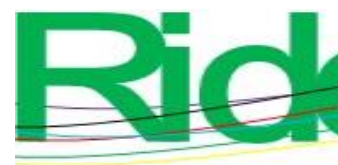
Revista Iberoamericana para la
Investigación y el Desarrollo Educativo
ISSN $2007-7467$

\section{Resumen}

Este artículo se centra en analizar los efectos que ha generado la pandemia por la covid-19 en la impartición de los cursos de matemáticas en la Universidad Autónoma de Nuevo León (UANL), máxima casa de estudios del norte de México. Para tal fin, se aplicó una encuesta en línea a 748 educandos de licenciatura en el segundo trimestre del año 2020. Con la información recabada se empleó un modelo Tobit censurado por la derecha, el cual permitió cuantificar el rendimiento académico de los estudiantes antes y durante el confinamiento en sus cursos o asignaturas de matemáticas en esta macrouniversidad. Entre los principales resultados se encuentra que la aplicación de software y plataformas digitales en la impartición de los cursos de matemáticas durante la contingencia sanitaria incrementa la eficiencia de los educandos en la solución y culminación de los ejercicios y los laboratorios, encargados por los docentes, en $23.2 \%$, mientras que el tiempo promedio para llevarlos a cabo es de 25.4 y 50.8 minutos, respectivamente.

Palabras clave: covid-19, software matemático, tobit.

\section{Abstract}

This article focuses on analyzing the effects that the COVID-19 pandemic has generated in the delivery of mathematics courses at the Autonomous University of Nuevo León (UANL), the highest house of studies in northern Mexico. To this end, an online survey is applied to 748 undergraduate students in the second quarter of 2020. With the information collected, a Tobit model censored by the right is used, which allows quantifying the academic performance of students before and during confinement in their math courses or subjects at this macro university. Among the main results is the application of software and digital platforms in the teaching of mathematics courses during the health contingency, increasing the efficiency of the students in the solution and completion of the exercises and the laboratories, commissioned by the teachers, in a $23.2 \%$, while the average time to carry out a line of 25.4 and 50.8 minutes, respectively.

Keywords: covid-19, mathematical software, tobit. 


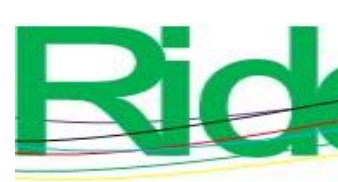

Revista Iberoamericana para la Investigación y el Desarrollo Educativo ISSN $2007-7467$

\section{Resumo}

Este artigo se concentra na análise dos efeitos que a pandemia do COVID-19 gerou na realização de cursos de matemática na Universidade Autônoma de Nuevo León (UANL), a mais alta casa de estudos no norte do México. Para isso, é aplicada uma pesquisa on-line a 748 estudantes de graduação no segundo trimestre de 2020. Com as informações coletadas, é utilizado um modelo Tobit censurado à direita, que permite quantificar o desempenho acadêmico dos alunos antes e durante confinamento em seus cursos ou disciplinas de matemática nesta macro universidade. Entre os principais resultados está a aplicação de software e plataformas digitais no ensino de cursos de matemática durante a contingência em saúde, aumentando a eficiência dos alunos na solução e conclusão dos exercícios e laboratórios, encomendados pelos professores, em uma 23,2\%, enquanto o tempo médio para realizar uma linha de 25,4 e 50,8 minutos, respectivamente.

Palavras-chave: covid-19, software matemático, tobit.

Fecha Recepción: Marzo 2021

Fecha Aceptación: Septiembre 2021

\section{Introducción}

La pandemia de la covid-19 ha afectado negativamente a la sociedad y parece que sus efectos se mantendrán, al menos, durante el año 2022. De acuerdo con datos de la Unesco (Organización de las Naciones Unidas para la Educación, la Ciencia y la Cultura, 2020), 188 países -incluido México - han implementado el cierre de escuelas y universidades como respuesta ante la pandemia, lo cual significa que más de $90 \%$ de los estudiantes en el mundo se encuentran sin asistir a clases presenciales.

Al respecto, Aquino y Medina (2020) explican que el aislamiento y la inmovilización social como medidas para disminuir el contagio de dicha enfermedad han obligado al cierre temporal de las instituciones de educación en todos los niveles. En el mismo sentido, Sanz, Sainz y Capilla (2020) señalan que este evento impredecible trajo consigo la suspensión abrupta de actividades administrativas, académicas y de investigación en todas las universidades, lo que obligó a implementar estrategias digitales para seguir con sus funciones de enseñanza y aprendizaje.

Ante esta problemática, la Universidad Autónoma de Nuevo León (UANL) ha optado por la educación virtual a distancia como respuesta inmediata a la covid-19 con la finalidad de no dejar de atender a la comunidad universitaria y evitar que se pierda el semestre académico. Sin duda alguna, la implementación de las clases y de los cursos virtuales han representado un reto para las 


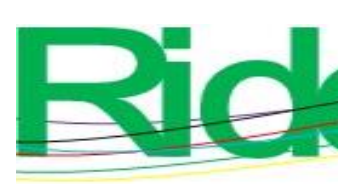

Revista Iberoamericana para la Investigación y el Desarrollo Educativo ISSN 2007 - 7467

autoridades, los administrativos, los docentes y los estudiantes de la UANL debido a ciertas limitantes que han surgido, como la disponibilidad de internet, la dificultad en el uso de plataformas virtuales, la capacitación al docente para cursos digitales, los ambientes inapropiados para la toma de cursos en el hogar, la saturación del soporte tecnológico, entre otras.

Pero ¿qué pasa con la impartición de los cursos de matemáticas?, ¿su enseñanza debe ser similar a la de materias teóricas? A partir de estas interrogantes, la presente investigación pretende analizar el efecto del confinamiento sobre el rendimiento académico de los estudiantes en sus cursos o asignaturas relacionadas con el área de matemáticas, específicamente en la culminación de los ejercicios y laboratorios propuestos por parte de los docentes de la UANL ${ }^{1}$.

Este trabajo parte de la premisa de que existe una relación positiva entre el uso de estrategias digitales, basadas en paquetes o software matemáticos, y la obtención de un mejor rendimiento académico en las asignaturas que contienen matemáticas, puesto que se considera que su empleo aumentan significativamente el aprendizaje y el desarrollo de habilidades relacionadas a este tipo de cursos.

Al respecto, Ruiz y Quintana (2016) sostienen que la enseñanza ortodoxa en matemáticas se ha visto cuestionada en los últimos años en relación con la calidad y la enseñanza debido a que los estudiantes revelan un bajo rendimiento en la solución y el entendimiento de problemas reales. De estas falencias no resulta extraño que se originen problemas de retraso escolar, deserción, reprobados, rechazo a las unidades de aprendizaje, desmotivación en el área, etc. Por esta razón, la presente investigación procura hilar más finamente dicho tema, al valerse de las capacitaciones virtuales otorgadas a los docentes de la UANL en técnicas y herramientas didácticas para el mejor aprovechamiento de los programas analíticos de las asignaturas en matemáticas en la modalidad en línea.

La hipótesis que se desea contrastar empíricamente es que el estudio o la enseñanza de los cursos relacionadas a las matemáticas son más eficientes y prácticos con la combinación de plataformas digitales y el uso de software matemáticos. Al momento de cuantificar el rendimiento académico de los educandos, en esta investigación se centra el análisis en los estudiantes de licenciatura y en los impactos que este cambio digital influye en la formación, la solución y la culminación de ejercicios y laboratorios impartidos en clase. Para ello, se han propuesto dos

\footnotetext{
${ }^{1}$ El término ejercicios, en este trabajo, hace referencia a aquellos problemas o actividades matemáticas desarrolladas en tiempo real dentro de la asignatura o curso, ya sea antes (clases presenciales) o durante la contingencia sanitaria (clases virtuales), mientras que los laboratorios son las tareas, ejercicios o problemarios matemáticos que son dejados por parte de los docentes para la solución en el hogar, ambos tomados para el semestre de enero a junio de 2020.
} 


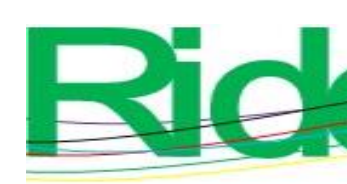

Revista Iberoamericana para la Investigación y el Desarrollo Educativo ISSN $2007-7467$

hipótesis particulares. A nivel individual, se cree que la comprensión y el entendimiento de los ejercicios propuestos en los cursos digitales — en tiempo real- impacta positivamente en la culminación y seguridad de los estudiantes en la solución de los problemas en menor tiempo; mientras que con los laboratorios se espera un mayor nivel de involucramiento con el uso de software matemáticos y su culminación será en un tiempo y una forma acordes al semestre.

Para lograr el objetivo de la investigación y dar respuesta a las hipótesis planteadas se implementó un cuestionario en línea, del cual se obtuvo respuesta de 748 educandos de diferentes disciplinas. Se aplica un modelo Tobit censurado por la derecha, el cual permite explicar el rendimiento académico (entendiéndose en este artículo como el tiempo que les lleva a los alumnos la solución o el término de los ejercicios y laboratorios en minutos) en función de los datos recabados antes (primeros meses del semestre, cuando los alumnos asistían a clases presenciales) y durante (últimos meses del semestre, cuando los estudiantes tomaban clases virtuales) el confinamiento en la macrouniversidad.

El trabajo se organiza como sigue: en la segunda sección se desarrolla una breve revisión teórica; en la sección tres se describen la metodología y la obtención de los datos; en la cuarta se reportan los resultados y, por último, se presentan algunas conclusiones y recomendaciones.

\section{Revisión de literatura}

Arteaga y Cruz (2018), Lamana y De la Peña (2018) y Medina, Fereira y Marzol (2018) señalan que existen diferentes causas que ocasionan bajo rendimiento académico en el área de matemáticas, entre las más comunes se encuentran las estrategias o técnicas didácticas ortodoxas (o muy obsoletas) por parte de los docentes, las cuales hacen que los educandos aprendan de manera mecánica los conceptos y fórmulas, lo que lleva al estudiante a preocuparse solo por aprobar el curso o la asignatura en lugar de adquirir un aprendizaje para su formación profesional.

Por su parte, Forés, Sánchez y Sancho (2014) sostienen que la principal razón del desinterés de los estudiantes a los cursos de matemáticas son los medios o técnicas didácticas rudimentarias que los docentes emplean en la enseñanza, pues les cuesta salir de su zona de confort o statu quo. Es decir, emplean los mismos métodos desde décadas pasadas, con los cuales se generan resultados desagradables para las nuevas generaciones de estudiantes.

Sin embargo, en estos tiempos de pandemia, la enseñanza en matemáticas en línea debe fortalecerse y mejorarse. Con la suspensión de las clases presenciales, los docentes deben ser creativos para ofrecer un aprendizaje significativo e interesante para los estudiantes. Aunque estos 


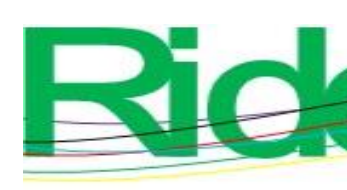

Revista Iberoamericana para la Investigación y el Desarrollo Educativo ISSN 2007-7467

últimos no estén disponibles físicamente para realizar los procedimientos y soluciones de ejercicios a problemas de matemáticas, los docentes de la UANL deben aprovechar los cambios que se están presenciando como sociedad en la actualización y la capacitación de enseñanza en el área de matemáticas para adaptarse a la contingencia sanitaria de la covid-19; por ejemplo, el uso de programas matemáticos en combinación de plataformas virtuales, lo que trae consigo clases en forma de tutoriales, considerados hasta hace poco un tabú en el sistema educativo, aunque en la actualidad la mayoría de los cursos académicos se están trasladando a un entorno en línea (Díaz, Vázquez, Olguín y Arau, 2018).

Asimismo, Ferrando, Segura y Pla (2017) destacan que involucrar a los estudiantes y a los profesores ante un cambio o modalidad de enseñanza-aprendizaje es desafiante, pero no imposible. Basta con conocer las herramientas y métodos de enseñanza innovadores en el campo de las matemáticas para que se obtengan resultados positivos y significativos en el desarrollo y formación de los estudiantes.

Por ello, el enfoque de esta sección es mostrar un marco de referencia sobre los modelos didácticos que comprenden la relación que existe entre los docentes y los estudiantes sobre la comunicación y el aprendizaje en estos tiempos de confinamiento. Al respecto, Alvarado, Morales y Téllez (2016), Alvarado, Luyando y Picazzo (2015) y Pari (2014) señala que un sistema educativo puede ser diferente según las condiciones del entorno del estudiante (infraestructura, planta docente, medios de enseñanza, desarrollo integral, entre otros). Por tal motivo, cualquier sistema académico debe ser capaz de tener un equilibrio entre el entorno académico (actualización de los medios de enseñanza y malla curricular de las asignaturas ante un mundo cambiante) y moral de los educadores (adaptación de la enseñanza y la evaluación flexible hacia a los educandos).

En lo que respecta a los modelos didácticos en la enseñanza y el aprendizaje de las matemáticas, Mayorga y Madrid (2010) establecen cuatro modelos con los que señalan las acciones planificadas por los educadores con la finalidad de que los alumnos alcancen su formación. El modelo didáctico tradicional explica la relación disciplinaria o rigurosa entre los educadores y su programa analítico de la asignatura por cubrir; en este modelo no se toman en cuenta las ideas de los estudiantes, pues los docentes deben seguir un estricto calendario de actividades y de pruebas para obtener una nota final (tareas, ejercicios, investigaciones y exámenes). En cambio, en el modelo didáctico alternativo se toman en consideración las ideas de los alumnos y se redireccionan en metodologías o estudios de caso basados en problemas reales o del entorno al cual están inmersos los estudiantes. El modelo didáctico tecnológico postula la combinación de experiencia 

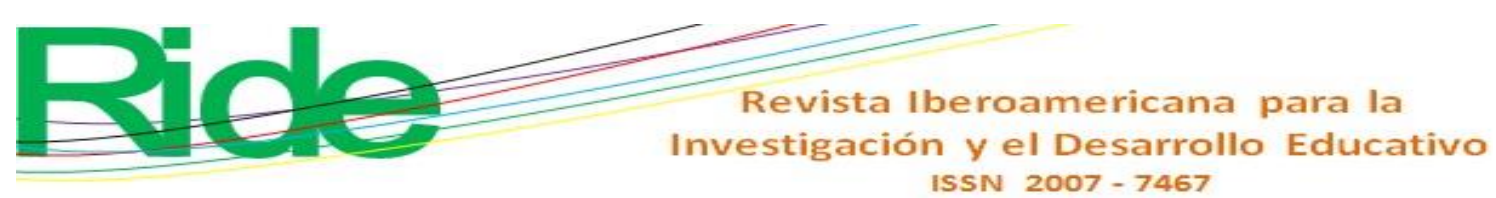

por parte del docente y el uso de herramientas tecnológicas o digitales con la finalidad de realizar un curso práctico, aplicado e interesante para los estudiantes. Por último, el modelo de didáctico activista se basa en el proceso de asimilación que los educandos obtienen, con base en contenidos o experiencias que los mismos alumnos han aprendido, vivido o desarrollado en el mercado laboral o en el entorno en el cual se desenvuelven.

En consecuencia, los cuatro modelos descritos muestran que la enseñanza y el aprendizaje, para el caso específico de las matemáticas, se basan en la obtención de conocimiento — sin importar que pase de lo abstracto a lo específico o de lo general a lo particular - con la finalidad de obtener un pensamiento reflexivo y crítico para impactar en la formación integral de los alumnos. De esta manera, se cree que el modelo didáctico tecnológico es el más acorde respecto a lo que sucede en la UANL, puesto que se espera — dada la contingencia de la covid-19- que los cursos de matemáticas se actualicen y se perfeccionen con la aplicación de software y plataformas tecnológicas con el objetivo de involucrar y acercar más a los estudiantes con el saber matemático.

Por último, en la siguiente sección se muestran aquellos estudios empíricos realizados sobre la innovación y la aplicación de tecnología en el proceso de la enseñanza en el área de matemáticas. Es conveniente mencionar que de los trabajos descritos a continuación son pocos los relacionados con la aplicación robusta de un modelo Tobit censurado por la derecha debido a que la literatura correspondiente hace referencia a investigaciones documentales, descriptivas y correlaciónales de las estrategias de enseñanza-aprendizaje en el tema que compete. De ahí el interés por hacer un aporte a este tipo de temas desde un enfoque cuantitativo.

\section{Importancia de la enseñanza-aprendizaje en matemáticas con herramientas tecnológicas}

El estudio de herramientas tecnológicas, métodos informáticos y simulación matemática a través de software o paquetes especializados está cobrando una importancia muy peculiar en los sistemas educativos. Meza y Cantarell (2002) y Gamboa (2007) señalan que, a partir del año 2000, el mundo está lleno de experiencias digitales y que las tecnologías proveen beneficios y oportunidades educativas a las personas que desean tener una formación académica completa. Por su parte, Haydel y Roeser (2002) argumentan que los estudiantes denominados millenials demuestran una clara preferencia por utilizar dispositivos móviles y tecnologías de información en el aula de clases. Adicionalmente, Molina (2017) y Fernández, Riveros y Montiel (2017) establecen 


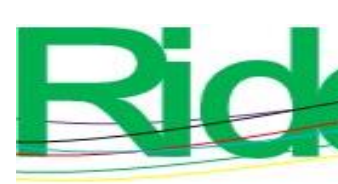

Revista Iberoamericana para la Investigación y el Desarrollo Educativo ISSN $2007-7467$

la motivación como factor esencial para el aprendizaje de asignaturas de matemáticas, con el uso de software como recurso indispensable en el área.

Ante la diversidad de herramientas tecnológicas es importante reconocer cuáles son las alternativas que se adaptan más al área de las matemáticas. Al respecto, Falck, Kluttig y Peirano (2013) sostienen que los educadores deben identificar cinco factores al momento de innovar una solución tecnológica en el aula. El primero se relaciona con las competencias individuales (necesarias por parte de los docentes y estudiantes para el uso de las tecnologías); en segundo lugar se encuentran las innovaciones para impartir el conocimiento (deben ser capaces de motivar e involucrar la participación de los educandos en clase); en tercer lugar, es necesario que la implementación tecnológica sea de la época o actuales (para que aporten un apoyo real al entorno del estudiante); en cuarto lugar, deben ser accesibles (para que los estudiantes no paguen demasiado por su uso); finalmente, es necesario que el programa o software tenga eficiencia y calidad en sus estimaciones.

En el mismo sentido, Almaguel, Álvarez, Pernía, Mota y Coello (2016), Avendaño, Rangel Ibarra y Chao González (2011) y Grisales (2018) destacan que el uso de software en clases de matemáticas favorece los procesos inductivos y la visualización de los conceptos en los estudiantes; además, permite comparar problemas y refutar hipótesis más prácticas y dinámicas, y sirve como motivación en el aprendizaje de contenidos matemáticos. A su vez, Zermeño, Navarrete y Contreras (2020) agregan que los programas o el uso de herramientas tecnológicas les permiten a los estudiantes desarrollar eficientemente sus habilidades y esfuerzos en la solución de problemas relacionados a las asignaturas de matemáticas y a su desarrollo humano.

Por su parte, Acosta, Mejía y Rodríguez (2011), Fernández, Izquierdo y Lima (2000), Díaz y Hernández (2002), Morales, Valencia, Martínez y Mario (2013), Gómez, Aguileta, Gómez y Aguilar (2018), Ruiz y Del Rivero (2019) y Salas (2018) establecen que el uso de software (CabriGeometric, Calculus, Derive, Eviews, FunReal, Mathematica, Modelus, SPSS, PSP, Stata, R, entre otros) ayuda a los estudiantes en la formación de conceptos, ejercitación y resolución de problemas. Su aplicación permite a los educandos ser más proactivos y participativos en el entendimiento y la comprensión de conocimientos de métodos cuantitativos.

Sin embargo, autores como Dede (2000) y Guédez (2005) indican que hay que recordar que el uso constante de tecnología no es la completa solución a todos los problemas o los contenidos de las asignaturas en matemáticas, puesto que el valor o el uso de las herramientas tecnológicas 


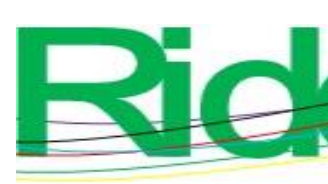

Revista Iberoamericana para la Investigación y el Desarrollo Educativo ISSN 2007 - 7467

está en función del diseño y la programación de las unidades de aprendizaje que los educadores desarrollan en clase.

Todas las investigaciones revisadas en este apartado, independientemente de las herramientas o plataformas tecnológicas que analizan, coinciden en que en la innovación y en la transformación que se da en el aula de clases es necesario que los docentes consideren adaptar su modelo didáctico de enseñanza hacia los estudiantes. Es decir, los educadores deben implementar una dinámica presencial tradicional, enriquecida tecnológicamente hacia un modelo didáctico digital.

En esta revisión de literatura no se encuentra evidencia de trabajos que hayan realizado un análisis empírico con un modelo Tobit censurado por la derecha, entre grupos de estudiantes, para cuantificar el término o culminación de ejercicios y laboratorios ante una etapa de confinamiento. Por esta razón, la presente investigación pretende cubrir el vacío de este tipo de análisis en esta temática, dada la situación que se presenta en la UANL ante la contingencia sanitaria de la covid19.

\section{Metodología}

Para cuantificar la caracterización y el rendimiento académico de los estudiantes universitarios en las asignaturas relacionadas al área de matemáticas ante el confinamiento en la UANL, se diseñó una encuesta en línea, disponible para todos los estudiantes vigentes de los campus universitarios durante el segundo trimestre del año 2020. Para ello, se realizó un muestreo no probabilístico por bola de nieve. ${ }^{2}$ En total, se recabaron 748 encuestas en dos grados de formación (licenciatura e ingeniería), que de manera virtual están tomando cursos o asignaturas relacionadas al campo de las matemáticas en el semestre entre enero y junio de 2020.

El cuestionario que se aplicó estuvo constituido por 21 preguntas, que se clasificaron en tres rubros disponibles en https://www.observatoriocedeem.com.mx/encuesta/. El primer rubro se enfocó en las características generales de los estudiantes (edad, género, semestre, carrera y facultad), mientras que el segundo se centró en los principales problemas que enfrentan los estudiantes ante la contingencia de la covid-19 (educativas, logísticas, tecnológicas y

\footnotetext{
${ }^{2}$ El muestreo por bola de nieve se utiliza para acceder a personas de baja incidencia o poblaciones de difícil acceso por parte del investigador. En este caso, la población objetivo estuvo constituida por todos aquellos estudiantes que se encontraban en confinamiento por la situación de la pandemia de covid-19.Por eso, se recopiló una base de correos electrónicos de estudiantes vigentes de los miembros del cuerpo académico UANL-CA-386 (del cual pertenecen los autores), y se les solicitó que contestaran la encuesta y recomendaran a un compañero de clase para su contestación.
} 


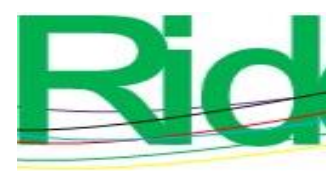

Revista Iberoamericana para la Investigación y el Desarrollo Educativo ISSN 2007 - 7467

Tabla 1: Descriptivos de las variables sociodemográficas y explicativas

\begin{tabular}{|c|c|c|c|c|c|}
\hline & \multirow{2}{*}{ Componente } & \multicolumn{4}{|c|}{ Estadísticos } \\
\hline & & Frecuencia & Porcentaje & Media & $\begin{array}{c}\text { Desviación } \\
\text { típica }\end{array}$ \\
\hline \multirow{3}{*}{ Género } & (1) Hombre & 469 & 62.7 & \multirow{3}{*}{0.63} & \multirow{3}{*}{0.484} \\
\hline & (0) Mujer & 279 & 37.3 & & \\
\hline & Total & 748 & 100.0 & & \\
\hline \multirow{5}{*}{ Edad } & (1) Menos de 20 años & 180 & 24.1 & \multirow{5}{*}{1.98} & \multirow{5}{*}{0.761} \\
\hline & (2) De 21 a 25 años & 450 & 60.2 & & \\
\hline & (3) De 26 a 30 años & 73 & 9.8 & & \\
\hline & (4) Más de 31 años & 45 & 6.0 & & \\
\hline & Total & 748 & 100.0 & & \\
\hline \multirow{4}{*}{ Área } & (1) Ciencias sociales & 523 & 69.9 & \multirow{4}{*}{1.36} & \multirow{4}{*}{0.586} \\
\hline & (2) Ingenierías & 184 & 24.6 & & \\
\hline & (3) Física y matemáticas & 41 & 5.5 & & \\
\hline & Total & 748 & 100.0 & & \\
\hline \multirow{5}{*}{ Semestre } & (1) De 1 a 3 semestres & 163 & 21.8 & \multirow{5}{*}{2.45} & \multirow{5}{*}{0.883} \\
\hline & (2) De 4 a 6 semestres & 122 & 16.3 & & \\
\hline & (3) De 7 a 9 semestres & 427 & 57.1 & & \\
\hline & (4) Más de 10 semestres & 36 & 4.8 & & \\
\hline & Total & 748 & 100.0 & & \\
\hline \multirow{5}{*}{ Problemas } & (1) Educativos & 29 & 3.9 & \multirow{5}{*}{3.12} & \multirow{5}{*}{0.984} \\
\hline & (2) Logísticos & 236 & 31.6 & & \\
\hline & (3) Tecnológicos & 102 & 13.6 & & \\
\hline & (4) Socioafectivos & 381 & 50.9 & & \\
\hline & Total & 748 & 100.0 & & \\
\hline \multirow{3}{*}{ Recursos } & (1) Comunicación & 323 & 43.2 & \multirow{3}{*}{1.57} & \multirow{3}{*}{0.496} \\
\hline & (2) Trabajo académico & 425 & 56.8 & & \\
\hline & Total & 748 & 100.0 & & \\
\hline \multirow{4}{*}{ Modalidad } & (1) Presencial & 401 & 53.6 & \multirow{4}{*}{1.76} & \multirow{4}{*}{0.878} \\
\hline & (2) Virtual & 128 & 17.1 & & \\
\hline & (3) Mixta & 219 & 29.3 & & \\
\hline & Total & 748 & 100.0 & & \\
\hline
\end{tabular}

Fuente: Elaboración propia 


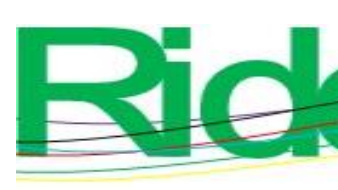

Revista Iberoamericana para la Investigación y el Desarrollo Educativo ISSN $2007-7467$

\section{Especificación del modelo empírico}

Como se menciona en la introducción, el objetivo de la presente investigación es cuantificar el rendimiento académico de los estudiantes antes y durante la contingencia sanitaria de la covid19. Para lograr lo anterior, las respuestas de los educandos se clasificaron en dos grupos, de modo que cada grupo contestó el tiempo promedio que dura en dar solución a los ejercicios y laboratorios antes y durante el confinamiento. Las respuestas que cada estudiante contestó sobre el tiempo que tardó en terminar sus ejercicios en clase (ya sea presencial o virtual) sirve para construir la variable minutos-ejercicios, mientras que los registros que tardan en terminar una tarea o problemario se le denomina minutos-laboratorios. Al mismo tiempo, en este procedimiento se identifica a los alumnos que, en el momento de la aplicación de la encuesta, no habían terminado sus ejercicios y laboratorios encomendados por los docentes, lo cual significa que se está ante un caso de datos censurados por la derecha. Por lo tanto, se opta por crear una variable de censura (censura) que vale 1 para los datos censurados y 0 para los no censurados.

Para llevar a cabo el ejercicio econométrico mencionado, se aplica un modelo Tobit censurado por la derecha (también conocido por censura superior o por encima), el cual permite obtener estimaciones cuando la variable dependiente (censura) no se observa para una parte de los educandos, es decir, es cero para una parte de los sujetos de estudio. Lo anterior puede representarse a través de un modelo Tobit:

$$
Y^{*}=X \beta+\mu \mu \mid X, c_{\sim} N\left(0, \sigma^{2}\right) Y=\min \left(Y^{*}, c\right)
$$

Que también puede escribirse:

$$
Y_{i}=\left\{\begin{array}{l}
c \text { si } Y_{i}^{*} \geq c \\
Y_{i}^{*} \text { si } Y_{i}^{*}<c
\end{array}\right.
$$

Dada la ecuación 2, se establece que $Y^{*}$ es menor que el valor de $c$, pero no se sabe cuánto vale; por lo tanto, se considera el modelo Tobit con cesura a la derecha. No obstante, para calcular las estimaciones de los parámetros hay que aplicar máxima verosimilitud (MV), puesto que en las observaciones sin censura $Y=Y^{*}$, la densidad de $Y$ es la misma que la de $Y^{*}$. Pero para las observaciones censuradas se tiene:

$$
P(Y=c \mid X)=P\left(Y^{*} \geq c \mid X\right)=P(u \geq c-X \beta \mid X=1-\varnothing[(c-X \beta) / \sigma](3)
$$

Posteriormente, se calcula el logaritmo de la función de verosimilitud y se realiza la estimación por MV con la finalidad de que $\operatorname{los} \beta_{j}$ se interpreten como un modelo clásico de regresión; para ello, la expresión del logaritmo de la función de verosimilitud para una muestra de tamaño como la del presente estudio es la siguiente (Pérez, 2005): 


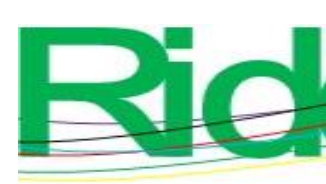

Revista Iberoamericana para la Investigación y el Desarrollo Educativo ISSN $2007-7467$

embargo, Hodges, Moore, Lockee, Trust y Bond (2020) apuntan que no es lo mismo responder a una catástrofe mundial al mostrar solidaridad y entusiasmo que incorporar en la cotidianidad las actividades a las que no se estaba acostumbrado, lo que deja futuras líneas de investigación en este fenómeno de estudio.

Es importante enfatizar que este trabajo pretende contribuir desde el punto de vista académico a los estudios y los análisis enfocados al proceso de enseñanza y asimilación en las matemáticas. Se evidencia con una muestra de corte transversal que para los estudiantes encuestados la modalidad virtual es efectiva en los cursos de matemáticas. En esta ocasión se realiza una investigación que busca probar, a través de un ejercicio econométrico, el rendimiento promedio de los educandos al terminar sus ejercicios y laboratorios en clases al utilizar herramientas tecnológicas, en donde se comprueba su eficiencia en el aprendizaje y la culminación en tiempos sobre los ejercicios y los laboratorios terminados en tiempo y forma.

Otros hallazgos interesantes en este documento fueron la identificación de los principales problemas que enfrentan los educandos ante el confinamiento por la covid-19 (en orden de importancia fueron los socioafectivos, tecnológicos, logísticos y educativos), así como observar los principales recursos tecnológicos que utilizan (Teams y Zoom). Estos resultados coinciden con Tyng, Amin, Saad y Malik (2017) y Zubieta, Bautista y Quijano (2012) al establecer que las herramientas tecnológicas buscan compartir información y conocimiento para interactuar con estudiantes, y que la comprensión y el entendimiento de las asignaturas sean efectivas y se consideren una alternativa para un futuro prometedor en los sistemas educativos.

\section{Conclusiones}

En esta investigación se demuestra que los estudiantes universitarios que llevan un curso o asignatura de matemáticas, en el semestre entre enero y junio de 2020 de la UANL, respondieron y se adaptaron positivamente a los software y plataformas digitales. De manera general, se encuentra que los educandos disminuyeron su tiempo de entrega y culminación en los ejercicios y laboratorios requeridos en los cursos de matemáticas.

Al utilizar el modelo Tobit censurado por la derecha, se controlan las respuestas otorgadas por los estudiantes por grupo de tiempos en la solución de los ejercicios y los laboratorios, antes y durante el confinamiento en la macrouniversidad, lo que da como principal resultado que los alumnos durante el confinamiento fueron los que cumplieron y concluyeron satisfactoriamente las actividades encomendadas en los cursos y asignaturas relacionadas al área de matemáticas. 


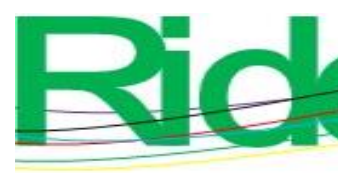

Revista Iberoamericana para la
Investigación y el Desarrollo Educativo
ISSN $2007-7467$

Por último, respecto al análisis descriptivo, se encuentra que las variables relacionadas con los problemas que enfrentan los estudiantes en esta contingencia son el estrés, la ansiedad y la frustración por el exceso de trabajo que presentan en el semestre, mientras que en los recursos tecnológicos afirmaron que utilizan más las plataformas Teams y WhatsApp para comunicarse con los docentes.

A partir de los hallazgos en este trabajo, se considera pertinente someter a consideración las siguientes recomendaciones académicas para enriquecer el conocimiento en el proceso de enseñanza en los educandos sobre el uso de software y plataformas digitales en los cursos y asignaturas de matemáticas como herramienta cognitiva, así como para mejorar la comprensión y enseñanza del tema en las matemáticas en los estudiantes: capacitación de manera conjunta entre docentes y alumnos para llevar a cabo acciones que garanticen el proceso de enseñanza-aprendizaje para todos.

Para lograr lo anterior, es necesario llegar a acuerdos con cuerpos académicos de la misma institución para que compartan su experiencia de investigaciones aplicadas a casos de estudios reales para el interés de los educandos. Asimismo, el apoyo institucional debe comprometerse a adquirir licencias de software o paquetes matemáticos, así como realizar cursos constantes de capacitación en herramientas, software y plataformas digitales para todos los actores involucrados en la enseñanza eficiente de las matemáticas.

\section{Futuras líneas de investigación}

Como trabajo futuro es pertinente continuar y dar seguimiento en el periodo inmediato posterior (semestres de 2022) con el objetivo de cuantificar las diferencias en la impartición de cursos de matemáticas después de la covid-19. Esto debido a que en el periodo de confinamiento (desde marzo de 2020) los docentes y alumnos han recibido capacitaciones orientadas a la creación de ambientes virtuales y software objeto de aprendizaje en la materia que han sido significativas y productivas en su formación académica. En este sentido, para futuros trabajos se recomienda ampliar el estudio a otras unidades de aprendizaje y universidades, de forma separada o simultánea. De igual modo, se sugiere ampliar la dimensión de este tópico, incluyendo un aumento en los participantes y facultades o departamentos de la universidad. También se debe considerar la implicación del fenómeno de estudio en los estudiantes de posgrado. 


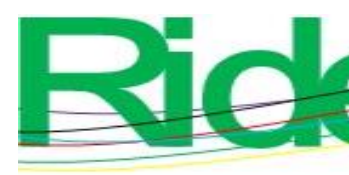

Revista Iberoamericana para la Investigación y el Desarrollo Educativo ISSN $2007-7467$

\section{Referencias}

Acosta, M., Mejía, C. y Rodríguez, C. (2011). Resolución de problemas por medio de matemática experimental: uso de software de geometría dinámica para la construcción de un lugar geométrico desconocido. Revista Integración, 29(2), 163-174.

Almaguel, A., Álvarez, D., Pernía, L., Mota, G. y Coello, C. (2016). Software educativo para el trabajo con matrices. Revista Digital Matemática, Educación e Internet, 16(2), 1-12.

Alvarado, E., Morales, D. y Téllez, E. (2016). Percepción de la calidad educativa: caso aplicado a estudiantes de la Universidad Autónoma de Nuevo León y del Instituto Tecnológico de Estudios Superiores de Monterrey. Revista de la Educación Superior, 45(180), 55-74.

Alvarado, E., Luyando, J. y Picazzo, E. (2015). Un análisis sobre la percepción que los estudiantes tienen de la calidad que ofrecen de las universidades privadas en Monterrey, Nuevo León. Revista Iberoamericana de Educación Superior, 6(17), 58-76.

Aquino, C. y Medina, C. (2020). Covid-19 y la educación en estudiantes de medicina. Revista Cubana de Investigaciones Biomédicas, 39(2), 1-4.

Arteaga, R. y Cruz, J. (2018). Factores incidentes en el rendimiento académico del área de estadística en estudiantes universitario. Polo del Conocimiento, 3(8), 281-291.

Avendaño, V., Rangel Ibarra, R. y Chao González, M. (2011). La enseñanza de las matemáticas en la realidad virtual. Revista de Tecnología y Sociedad, 1(1), 1-22.

Cuicas, M., Debel, C., Casadei, L. y Álvarez, Z. (2007). El software matemático como herramienta para el desarrollo de habilidades del pensamiento y mejoramiento del aprendizaje de las matemáticas. Revista Electrónica Actualidades Investigativas en Educación, 7(2), 1-34.

Dede, C. (2000). Aprendiendo con tecnología. España: Editorial Paidós Ibérica, S. A.

Díaz, A., Vázquez, I., Olguín, Z. y Arau, A. A. (2018). Enseñanza-aprendizaje a nivel posgrado con la aplicación Youtube. En Makita, T., Gaber, V., León, J. y Caballero, F. (coords.), Innovación educativa. Avances de cuerpos académicos en casos y aplicaciones (pp.117123). Red IBAI.

Díaz, F. y Hernández, G. (2002). Estrategias docentes para un aprendizaje significativo: una interpretación constructivista (2. ${ }^{\mathrm{a}}$ ed.). McGraw-Hill Interamericana.

Falck, D., Kluttig, M. y Peirano, C. (2013). TIC y educación. La experiencia de los mejores: Corea, Finlandia y Singapur. Editorial Santillana.

Fernández, F., Izquierdo, J. y Lima, S. (2000). Experiencias en la estructuración de clases de matemáticas empleando asistentes matemáticos y colección de tutoriales hipermediales. 


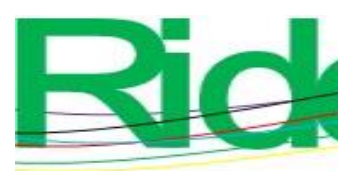

Revista Iberoamericana para la
Investigación y el Desarrollo Educativo
ISSN $2007-7467$

Medina, N., Fereira, J. y Marzol, R. (2018). Factores personales que inciden en el bajo rendimiento académico de los estudiantes de geometría. Telos, 20(1), 4-28.

Meza, A. y Cantarell, L. (2002). Importancia del manejo de estrategias de aprendizaje para el uso educativo de nuevas tecnologías de información y comunicación en educación. Ciberoteca Mística, 2-13.

Molina, J. A. (2017). Experiencia de modelación matemática como estrategia didáctica para la enseñanza de tópicos de cálculo. Uniciencia, 31(2), 1-22.

Morales, F., Valencia, A., Martínez, R. y Mario, J. (2013). Análisis de software matemático usados en nivel superior. Revista Vinculos, 10(1), 299-307.

Organización de las Naciones Unidas para la Educación, la Ciencia y la Cultura (UNESCO) (2020).

Impacto de covid-19 en educación. Recuperado de https://es.unesco.org/covid19/educationresponse

Oteiza, F. y Silva, H. (2001). El conocimiento matemático que se enseña en la escuela: ¿Está respondiendo a los nuevos desafíos de la educación? Centro Comenius USACH, 75, 1-18.

Pari, A. (2014). Modelos didácticos en las clases de educación física escolar. Revista Universitaria de la Educación Física y el Deporte, 7, 42-50.

Pérez, C. (2005). Métodos estadísticos avanzados con SPSS. Thomson Editores.

Ruiz, G. y Quintana, A. (2016). Atribución de motivación de logro y rendimiento académico en matemática. PsiqueMag, 4(1), 81-98.

Ruiz, L. y Del Rivero, S. (2019). Impacto de la matemática en el contexto de las ciencias con software matemático en ecuaciones diferenciales. Científica, 23(1), 13-21.

Salas, R. A. (2018). Uso del modelo TPACK como herramienta de innovación para el proceso de enseñanza-aprendizaje en matemáticas. Perspectiva Educacional, 57(2), 3-26.

Sanz, I., Sáinz, J. y Capilla, A. (2020). Efectos de la crisis del coronavirus en la educación superior. Organización de Estados Iberoamericanos para la Educación, la Ciencia y la Cultura (OEI). Recuperado de https://oei.org.br/arquivos/informe-covid-19d.pdf

Tyng, M., Amin, U., Saad, N. M. and Malik, S. (2017). The influences of emotion on learning and memory. Frontiers in Psychology, 8, 1-22.

Zermeño, A. I., Navarrete, M. y Contreras, I. L. (2020). En busca de los usos productivos de las TIC para el desarrollo humano de los jóvenes universitarios. Revista de Tecnología y Sociedad, 10(18), 1-23. 


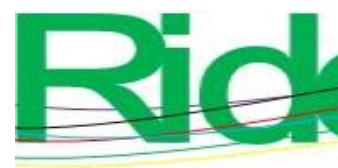

Revista Iberoamericana para la Investigación y el Desarrollo Educativo ISSN $2007-7467$

Zubieta, J., Bautista, T. y Quijano, A. (2012). Aceptación de las TIC en la docencia: una tipología de los académicos de la UNAM. Editorial Porrúa.

\begin{tabular}{|c|c|}
\hline Rol de Contribución & Autor (es) \\
\hline Conceptualización & $\begin{array}{l}\text { ELÍAS ALVARADO LAGUNAS, DIONICIO MORALES } \\
\text { RAMÍREZ (IGUAL). }\end{array}$ \\
\hline Metodología & ELÍAS ALVARADO LAGUNAS \\
\hline Software & $\begin{array}{l}\text { ELÍAS ALVARADO LAGUNAS, DIONICIO MORALES } \\
\text { RAMÍREZ (QUE APOYA). }\end{array}$ \\
\hline Validación & ELÍAS ALVARADO LAGUNAS \\
\hline Análisis Formal & $\begin{array}{l}\text { ELÍAS ALVARADO LAGUNAS, DIONICIO MORALES } \\
\text { RAMÍREZ (IGUAL). }\end{array}$ \\
\hline Investigación & JEYLE ORTIZ RODRÍGUEZ \\
\hline Recursos & JEYLE ORTIZ RODRÍGUEZ \\
\hline Curación de datos & $\begin{array}{l}\text { ELÍAS ALVARADO LAGUNAS, JEYLE ORTIZ } \\
\text { RODRÍGUEZ (IGUAL). }\end{array}$ \\
\hline $\begin{array}{l}\text { Escritura - Preparación del } \\
\text { borrador original }\end{array}$ & $\begin{array}{l}\text { ELÍAS ALVARADO LAGUNAS, JEYLE ORTIZ } \\
\text { RODRÍGUEZ (IGUAL). }\end{array}$ \\
\hline $\begin{array}{l}\text { Escritura - Revisión y } \\
\text { edición }\end{array}$ & $\begin{array}{l}\text { ELÍAS ALVARADO LAGUNAS, JEYLE ORTIZ } \\
\text { RODRÍGUEZ (QUE APOYA). }\end{array}$ \\
\hline Visualización & ELÍAS ALVARADO LAGUNAS \\
\hline Supervisión & JEYLE ORTIZ RODRÍGUEZ \\
\hline Administración de Proyectos & $\begin{array}{l}\text { ELÍAS ALVARADO LAGUNAS, DIONICIO MORALES } \\
\text { RAMÍREZ (IGUAL). }\end{array}$ \\
\hline Adquisición de fondos & $\begin{array}{l}\text { ELÍAS ALVARADO LAGUNAS, DIONICIO MORALES } \\
\text { RAMÍREZ, JEYLE ORTIZ RODRÍGUEZ (IGUAL). }\end{array}$ \\
\hline
\end{tabular}

